\title{
Energy Band Analysis of MQW Structure Based on Kronig-Penny Model
}

\author{
Yu Zhang ${ }^{1 *}$, Yi Wang ${ }^{2}$ \\ ${ }^{1}$ School of Physics and Engineering, Sun Yat-sen University, Guangzhou, China \\ ${ }^{2}$ School of Applied Physics and Materials, Wuyi University, Jiangmen, China \\ Email: ${ }^{*}$ zykelly18@163.com, yiwangl1@yahoo.com.cn \\ Received May 1, 2013; revised June 2, 2013; accepted June 28, 2013
}

Copyright (C) 2013 Yu Zhang, Yi Wang. This is an open access article distributed under the Creative Commons Attribution License, which permits unrestricted use, distribution, and reproduction in any medium, provided the original work is properly cited.

\begin{abstract}
The effects of different potential well depths, well widths and barrier widths on energy band of multiple quantum well (MQW) structures are discussed in detail based on Kronig-Penny model. The results show that if the well and barrier width stay unchanged, the first and second band gaps increase linearly with the well depth. When the well depth is constant, the first and second band gaps increase exponentially with the barrier width in a wide well. However, in narrow well one, the second band gap saturates when the barrier width is wide enough. On condition that the well and barrier have equal width, the first band gap decreases exponentially with well-barrier width while the second gap still shows an exponential increase with the width. These results are insightful for the design of MQW structure optoelectronic devices.
\end{abstract}

Keywords: Kronig-Penny Model; MQW Structure; Potential Well; Barrier

\section{Introduction}

Recent years have seen the rapid development of lightemitting diodes (LEDs), laser diodes (LDs) and solar cells. Particularly, LEDs have become high-performance devices widely used in display and lighting industry [1]. The core of these devices is the MQW structure. So, it is obvious that researches concerning features of MQW energy band are the foundation of further development of LED, LD devices and so on. A major obstacle for GaInN based LED to further penetrate into the general illumination market is that their efficiency suffers a substantial decrease as the injection current increases, which is called "efficiency droop" [2-7]. Researches show that the decrease of the barrier height within the MQW region $[1,8]$, such as applying p-type-doped barriers or a lightly n-type-doped GaN injection layer just below the InGaN MQWs on the $n$ side [1,9] and the trapezoidal wells of MQW structure [10] lead to the reduction of efficiency droop at high injection levels. The GaInN/GaInN MQW structure has much lower triangular barriers in the active region which also contributes to the enhancement of LED performances [2]. The emission wavelength of InGaN-Based MQW structure LD is the shortest one ever generated by a semiconductor laser diode [11]. InGaN/

\footnotetext{
"Corresponding author.
}

GaN MQW can also improve the behavior of solar cells. $[12,13]$ In this way, a profound study of different MQW structure is essential. Kronig-Penney model (K-P model) has been widely used in analyzing the energy band behavior of crystals and super lattices [14,15]. Some researchers studied the spectrum, transmission and conductance of electrons in bilayer graphene with K-P model [16]. Further discussion concerning energy band behavior by K-P model is of great importance for understanding the band features and electron transmission behavior of MQW structure. However, there are few reports investigating comprehensively the quantum mechanics and energy band features of different MQW structure. In this article, the energy band behavior of MQW with different potential well depths, widths and barrier widths is discussed using Kronig-Penney model. With the aid of Mathematica, the efficiency of calculation and accuracy of theoretical analysis are enhanced.

\section{Kronig-Penney Model}

Kronig-Penney model [17] is a potential field model with periodic array of rectangular potential wells.

The schematic Kronig-Penney model of the crystal is shown in Figure 1. Assuming the width of potential well is $a$ and the barrier width is $b, V_{0}$ is the depth of quantum 

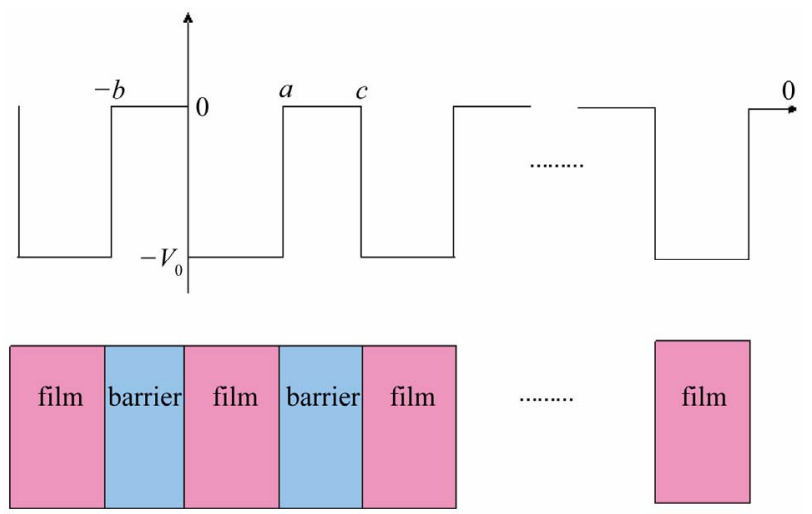

Figure 1. Kronig-penney model and real film made MQW structure.

well or the height of quantum barrier, the periodic potential field can be described as:

$$
V(x)=\left\{\begin{array}{l}
0, \quad n c+a<x<(n+1) c \\
-V_{0}, \quad n c<x<n c+a
\end{array}\right.
$$

where $c=a+b$ is the period of potential field.

The Hamiltonian of the system can be given as:

$$
H=-\frac{\hbar^{2}}{2 m} \frac{\mathrm{d}^{2}}{\mathrm{~d} x^{2}}+V(x)
$$

In well region $0<x<a$, the wave function is given by the superposition of two wave functions propagating toward left and right respectively, that is:

$$
\psi_{w}=A \mathrm{e}^{i K x}+B \mathrm{e}^{-i K x}
$$

where $K$ is limited by $\frac{\hbar^{2} K^{2}}{2 m}=E+V_{0}>0$

In barrier region $-b<x<0$, the wave function can be expressed as:

$$
\psi_{b 1}=C \mathrm{e}^{F x}+D \mathrm{e}^{-F x}
$$

with $F$ confined by $\frac{\hbar^{2} F^{2}}{2 m}=-E>0$.

According to Bloch theorem, the wave function $\psi_{b 1}$ in another barrier region $a<x<c$ can be expressed by $\psi_{b 2}$ as:

$$
\psi_{b 2}(x+c)=\mathrm{e}^{i k c} \psi_{b 1}(x)
$$

where $k$ is the electron wave vector.

A, B, C, D can be obtained by continuity requirements of wave functions and their first derivatives at well-barrier boundaries. The determinant constituted by the coefficients of A, B, C, D should be zero.

$$
\left|\begin{array}{cccc}
1 & 1 & -1 & -1 \\
i K & -i K & -F & F \\
\mathrm{e}^{i K a} & \mathrm{e}^{-i K a} & -\mathrm{e}^{i k(a+b)} e^{-F b} & -\mathrm{e}^{i k(a+b)} \mathrm{e}^{F b} \\
i K \mathrm{e}^{i K a} & -i K \mathrm{e}^{i K a} & -\mathrm{e}^{i k(a+b)} e^{-F b} F & \mathrm{e}^{i k(a+b)} \mathrm{e}^{F b} F
\end{array}\right|=0
$$

We can get the following equation by simplifying the determinant:

$$
\begin{aligned}
& \frac{F^{2}-K^{2}}{2 F K} \sinh F b \sin K a \\
& +\cosh F b \cos K a=\cos k(a+b)
\end{aligned}
$$

The left term of Equation (6) can be plotted without introducing infinite deep potential well approximation.

$$
\begin{aligned}
f(K) & =\frac{F^{2}-K^{2}}{2 F K} \sinh F b \sin K a \\
& +\cosh F b \cos K a
\end{aligned}
$$

where $F=\sqrt{\frac{2 m V_{0}}{\hbar^{2}}-K^{2}}$

The value of function $\cos k(a+b)$ on the right of Equation (6) ranges from -1 to 1 so that $K$ is available only when the value of $f(K)$ is between -1 to 1 . The graph of $f(K)$ is shown in Figure 2. AB stands for the width of the first energy gap and CD is the width of the second energy gap.

The influence of different well depths, well widths and barrier widths on energy band of MQW structure is investigated as following.

\section{Energy Band Features of Different MQW Structure}

\subsection{The Influence of Different Well Depths on Energy Band}

\subsubsection{Cases of Potential Well and Barrier with Equal Width}

On condition that the potential well and barrier have equal width, MQW structure with equal width of well and barrier, i.e. $a=b=0.5$, and well depth taking the value of $3,6,9,12$ separately are studied, where $a, b$, and $V_{0}$ are taken an arbitrary unit. The graphs of $f(K)$ vs $K$ are shown in Figure 3.

As shown in Figure 2, AB is the width of the first energy gap and $\mathrm{CD}$ is the width of the second energy gap.

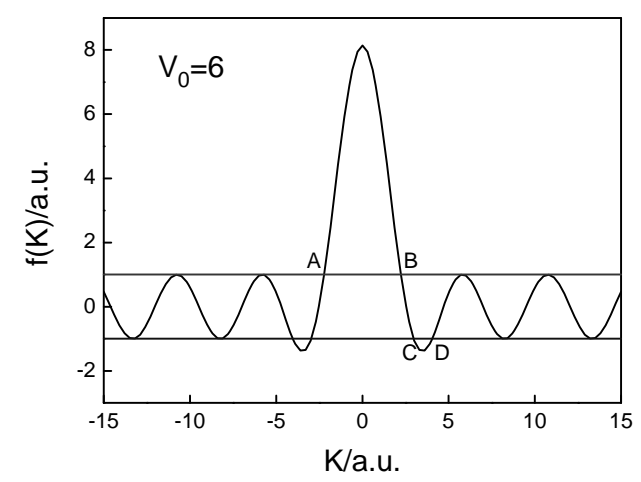

Figure 2. Energy band structure of multiple quantum wells $\left(a=b=0.6, V_{0}=6\right)$. 

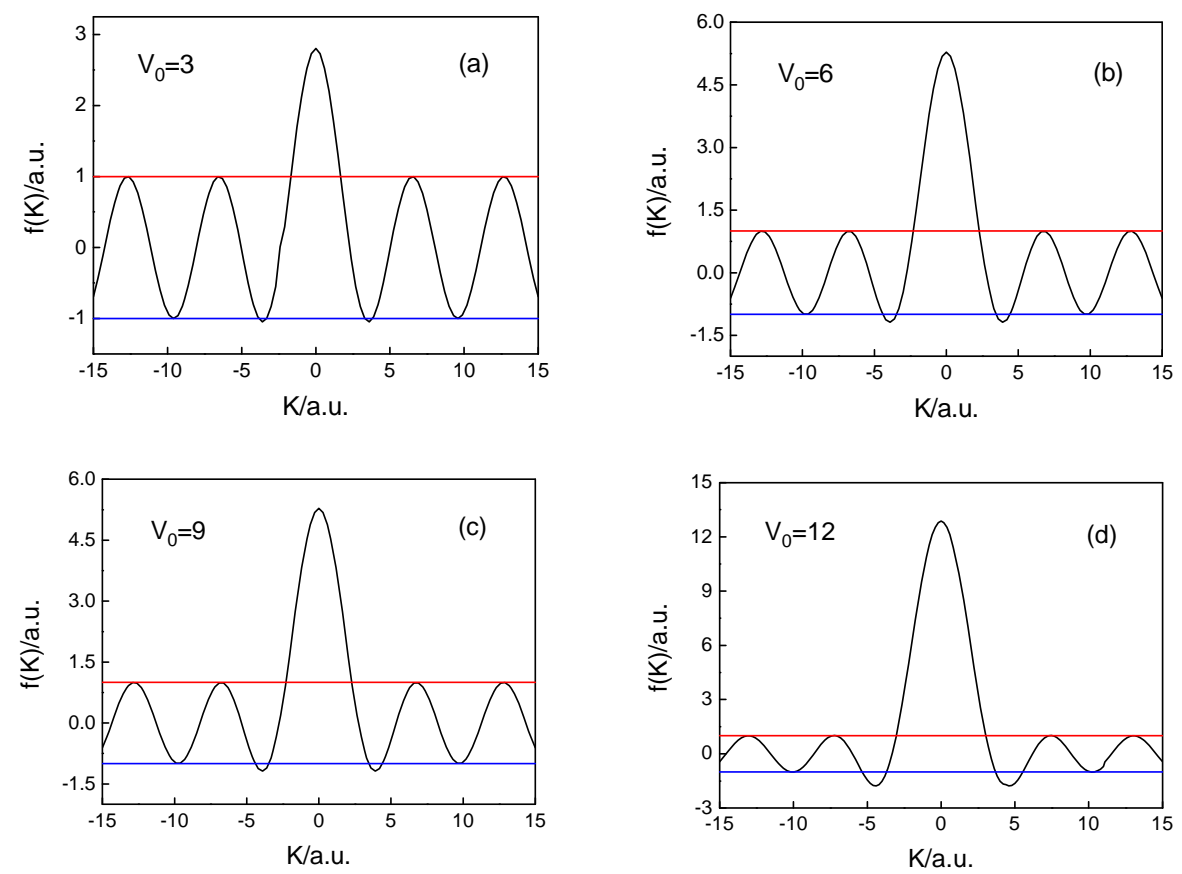

Figure 3. MQW structure with $a=b=0.5$. (a), (b), (c) and (d) correspond to well depth 3, 6, 9, 12 respectively.

The values of the first and second energy band gap in the four graphs of Figure 3 can be obtained according to the calculation, the results of which are given in Table 1.

Via linear fitting, the relationship of well depth and energy band gaps is obtained, as shown in Figure 4.

The relationship between the first energy band gap and well depth (Figure 4(a)) can be approximated to a linear function $y_{1}=0.3 x+2.62$ with linearly dependent coefficient of 0.9887 . The relationship between the second energy band gap and well depth (Figure 4(b)) can also be approximated by a linear function $y_{2}=0.13 x+0.19$ with linearly dependent coefficient of 0.9979 . It can be concluded that the first and second energy band gaps increase linearly with the well depth when the well width equals to the barrier width.

\subsubsection{Cases of Potential Well and Barrier with Unequal Width}

MQW structure devices with different well width and barrier width are an ubiquitous situation in practical applications. Discussing the situation of well width differing from barrier width can offer theoretical instructions for LED, solar cell designing and study of new materials, like graphene. Here, MQW structure with well width $a=$ 0.8 , barrier width $b=0.2$ is taken into consideration. The change of the first and second energy band gap values with well depth is shown in Table 2.

As shown in Figure 5, the relationship between the first energy band gap and well depth (Figure 5(a)) can be approximated by a linear function $y_{1}=0.21 x+1.64$ with linearly dependent coefficient of 0.98991 ; and the
Table 1. The changes of the first and second energy band gap values with well depth $(a=b=0.5)$.

\begin{tabular}{lcccc}
\hline Well depth & 3 & 6 & 9 & 12 \\
$\begin{array}{l}\text { The first energy } \\
\text { band gap }\end{array}$ & 3.35460 & 4.58822 & 5.43148 & 6.06226 \\
$\begin{array}{l}\text { The second energy } \\
\text { band gap }\end{array}$ & 0.53438 & 0.96853 & 1.34005 & 1.66706 \\
\hline
\end{tabular}
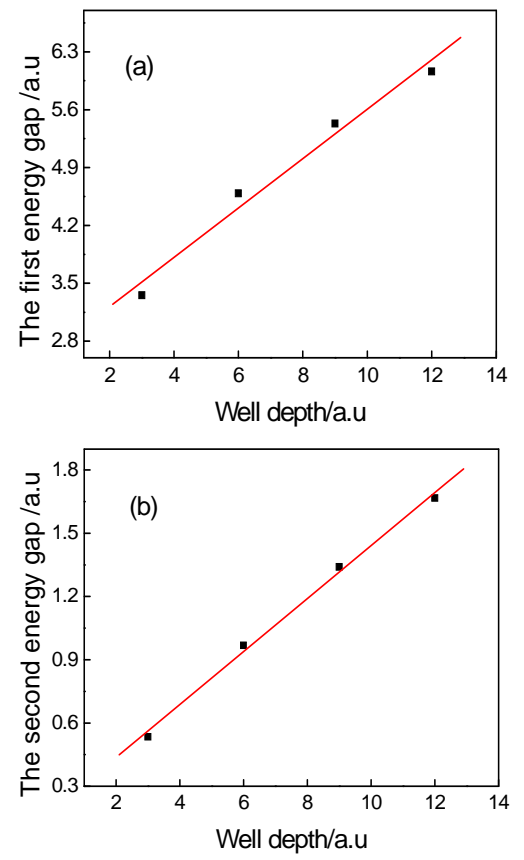

Figure 4. Linear fitting of well depth and energy band gaps with equal well and barrier width. (a) and (b) correspond to the first and second energy band gap respectively. 
Table 2. The first and second energy band gaps as the function of well depths $(a=0.8, b=0.2)$.

\begin{tabular}{lcccc}
\hline Well depth & 3 & 6 & 9 & 12 \\
$\begin{array}{l}\text { The first energy } \\
\text { band gap }\end{array}$ & 2.12240 & 2.90948 & 3.45674 & 3.87540 \\
$\begin{array}{l}\text { The second energy } \\
\text { band gap }\end{array}$ & 0.32891 & 0.61104 & 0.85702 & 1.07386 \\
\hline
\end{tabular}
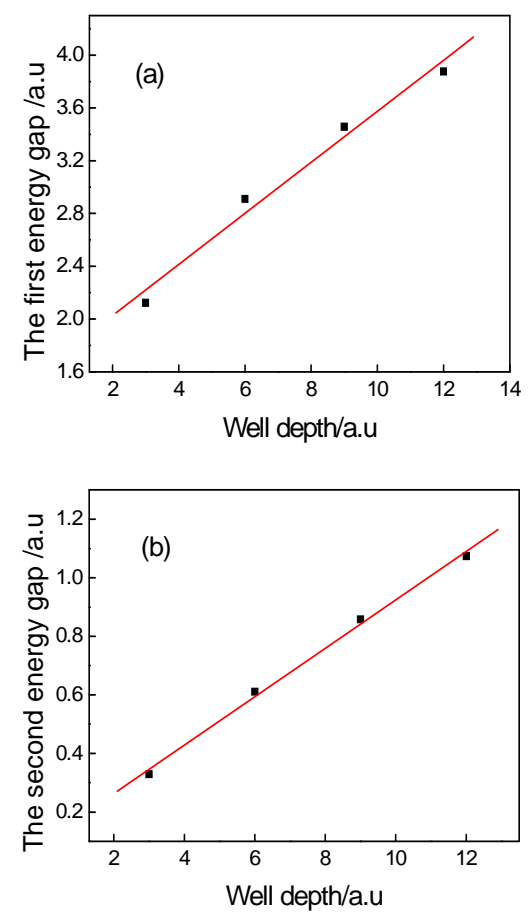

Figure 5. Linear fitting of well depth and energy band gaps when well width is unequal with barrier width. (a), (b) correspond to the first and second energy band gap respectively.

second energy band gap as the function of well depth (Figure 5(b)) can also be given as a linear function $y_{2}=0.10 x+0.10$ with linearly dependent coefficient of 0.99827 . The analyses and calculation reveal that the first and second energy band gaps increase linearly with the well depth in the case of unequal width of well and barrier.

According to the theoretical analyses above, we can see that the first and second band gaps increase linearly with the depth of quantum well if the well and barrier widths are kept constant. The result suggests that the deep potential well is equivalent to the case of narrow quantum well structure, in which the quantum mechanics effect is enhanced. For an infinite depth potential well, the quantum effect of the first level energy is getting enhanced, thus leads to the widening of the first energy band gap. Meanwhile, due to the increase of quantum tunneling effect, the quantum effect of the second level energy is also getting strengthened, and consequently leads to the widening of the second energy band gap.

\subsection{The Influence of Different Well and Barrier Widths}

\subsubsection{The Influence of Barrier Width on Wide Well MQW}

Wide potential well structure is the key feature for many microelectronic devices, such as solar cells $[12,18]$. In order to understand the mechanism of different MQW structure for a desired electronic device designing, it is important to investigate the wide well band features. For a MQW structure with well depth $V_{0}=6$ and well width $a=0.8$, barrier width $b$ varying from 0.2 to 1 , the energy gaps of the first band and second band are shown in Table 3.

The first and second energy band gaps as a function of barrier width are shown in Figure 6. The relationship between the first energy band gap and barrier width (Figure 6(a)) can be fitted to an exponential function $y_{1}=-2.71 \mathrm{e}^{-\frac{x}{0.32}}+4.35$; while the second energy band

Table 3. The change of the first and second energy band gaps with barrier width (well depth $V_{0}=6$, well width $a=$ 0.8).

\begin{tabular}{lccccc}
\hline Well width $a$ & 0.8 & 0.8 & 0.8 & 0.8 & 0.8 \\
$\begin{array}{l}\text { Barrier width } b \\
\begin{array}{l}\text { The first energy } \\
\text { band gap }\end{array}\end{array}$ & 0.2 & 0.4 & 0.6 & 0.8 & 1.0 \\
$\begin{array}{l}\text { The second energy } \\
\text { band gap }\end{array}$ & 0.61104 & 0.96156 & 1.14882 & 1.24543 & 1.29350 \\
\hline
\end{tabular}
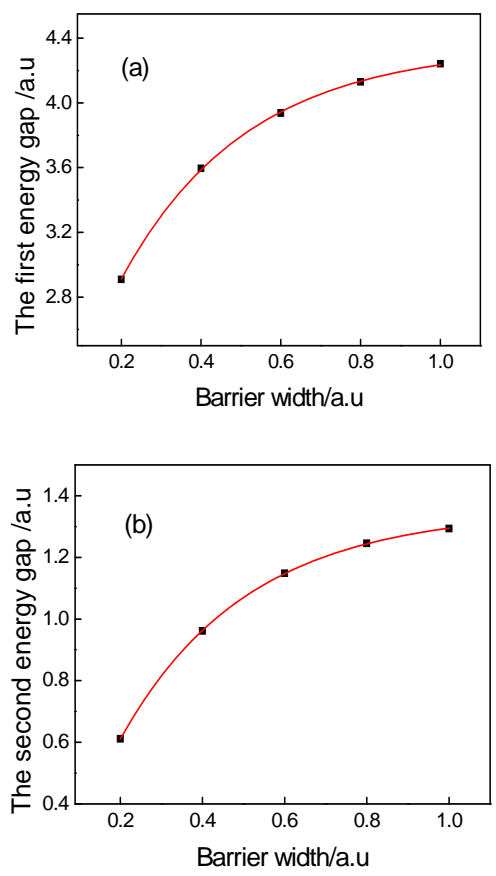

Figure 6. Energy gaps VS barrier width in a wide well MQW structure. (a), (b) correspond to the first and second energy band gap respectively. 
gap as a function of barrier width (Figure 6(b)) can be given as $y_{2}=-1.41 \mathrm{e}^{-\frac{x}{0.31}}+1.35$. It can be concluded that the first and second energy band gaps increase exponenttially with the barrier width in wide well MQW structure when the well depth is kept constant.

\subsubsection{The Influence of Barrier Widths on a Narrow Well MQW}

Narrow potential well MQW is also widely used in many electronic devices, such as LEDs [19]. For a narrow well MQW with well depth $V_{0}=6$ and well width $a=0.2$, the barrier width $b$ varying from 0.1 to 1 , the energy gaps of first band and second band are shown in Table 4.

Figure 7 demonstrates the relation between first and second energy band gaps and barrier width in a narrow well MQW structure. The relationship between the first energy band gap and barrier width (Figure 7(a)) can be approximated by an exponential function

Table 4. The first and second energy band gaps vs barrier width $\left(V_{0}=6, a=0.2\right)$.

\begin{tabular}{lccccc}
\hline Well width $a$ & 0.2 & 0.2 & 0.2 & 0.2 & 0.2 \\
$\begin{array}{l}\text { Barrier width } b \\
\begin{array}{l}\text { The first energy } \\
\text { band gap }\end{array}\end{array}$ & 0.1 & 0.2 & 0.3 & 0.4 & 0.6 \\
$\begin{array}{l}\text { The second energy } \\
\text { band gap }\end{array}$ & 0.30910 & 0.46453 & 0.53600 & 0.56535 & 0.56746 \\
\hline
\end{tabular}
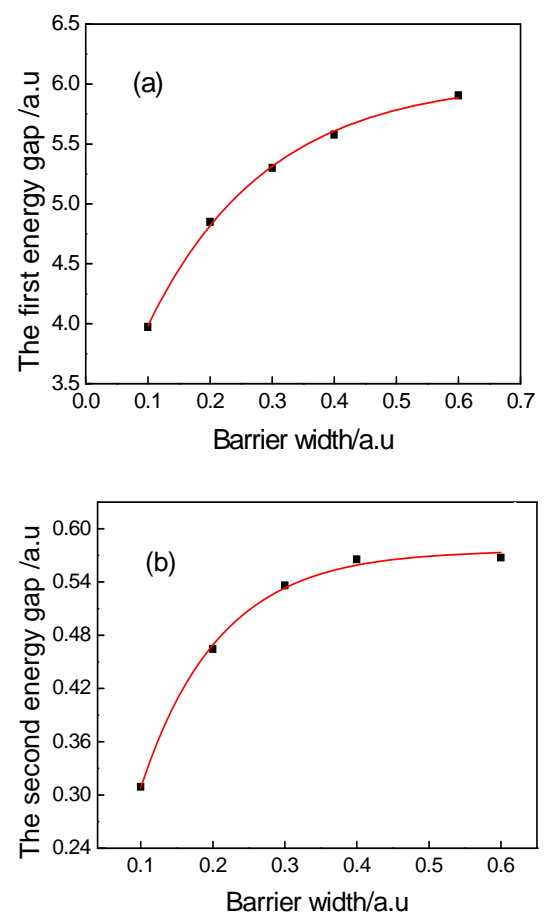

Figure 7. Energy band gaps of narrow well MQW vs barrier width. (a), (b) correspond to the first and second energy band gap respectively. $y_{1}=-3.46 \mathrm{e}^{-\frac{x}{0.19}}+6.04$; The relationship of the second energy band gap and barrier width (Figure 7(b)) can be described by an exponential function

$y_{2}=-0.67 \mathrm{e}^{-\frac{x}{0.11}}+0.58$. The graphs show that the first and second energy band gaps increase exponentially with the increase of barrier width in narrow well MQW structure when the well depth is kept constant. However, the second band gap value becomes saturated when the barrier width $b$ is comparatively large, and it no longer increases with the barrier width.

\subsubsection{The Influence of Equal Well-Barrier Width on Energy Band}

For a comprehensive understanding of band features for different MQW structures, the MQW structure with equal width well-barrier is also taken into consideration. For a certain well depth $V_{0}=6$, we assume the values of equal well-barrier width $(a=b=d)$ vary from 0.2 to 1 , the calculated results are given in Table 5.

Figure 8 presents the relationship between the first

Table 5. The first and second energy band gaps with equal well-barrier width $\left(V_{0}=6\right)$.

\begin{tabular}{lccccc}
\hline $\begin{array}{l}\text { Equal well-barrier } \\
\text { width }(d)\end{array}$ & 0.2 & 0.4 & 0.6 & 0.8 & 1.0 \\
$\begin{array}{l}\text { The first energy } \\
\text { band gap }\end{array}$ & 4.84976 & 4.70040 & 4.45296 & 4.12944 & 3.77426 \\
$\begin{array}{l}\text { The second energy } \\
\text { band gap }\end{array}$ & 0.46453 & 0.82983 & 1.08173 & 1.24543 & 1.34076 \\
\hline
\end{tabular}
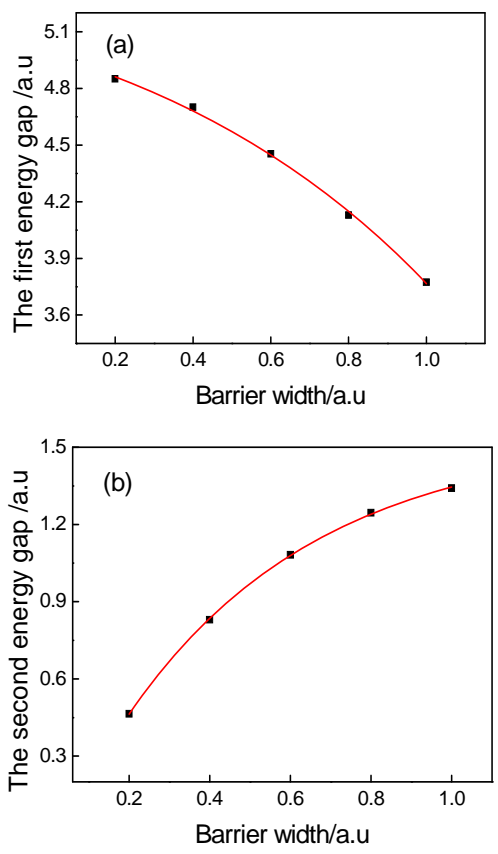

Figure 8. Energy band gaps as the function of equal wellbarrier width. (a), (b) correspond to the first and second energy band gap respectively. 
and second energy band gaps and the equal well-barrier width $d$. The first energy band gap as the functions of equal well-barrier width (Figure 8(a)) can be described as $y_{1}=0.5 \mathrm{e}^{-\frac{x}{0.18}}+5.51$, and the second relation (Figure 8(b)) can be given as function $y_{2}=-1.65 \mathrm{e}^{-\frac{x}{0.47}}+1.55$. It can be concluded from Figure 8 that the first energy band gap decreases exponentially with the well-barrier width $d$ while the second energy band gap still shows an exponential increase with the well-barrier width for aequilate MQW structure when the well depth is kept constant.

The increase of well-barrier width is equivalent to the diminution of well depth. It leads to weakened quantum effect and the decrease of the first energy band gap width. On the other hand, due to the diminution of well depth, the electrons leap across the barrier more easily, thus leading to the enhancement of correlating effect of electrons between the well and the barrier which further results in the broadening of the second band gap.

\section{Conclusion}

The energy band features of MQW with different well depths, well widths and barrier widths by K-P model are investigated in the paper. We found that, the first and second band gaps increase linearly with the increase of well depth when the well and barrier width stay unchanged. Under the condition that the well depth keeps constant, the first and second band gaps of wide potential well structure increase exponentially with the barrier width. While in a narrow well case, the second band gap becomes saturated when the barrier width is wide enough. If the well and barrier have equal width, the first energy band gap decreases exponentially with the well-barrier width while the second gap increases exponentially with the width. These conclusions present us some interesting band features of MQW structure with different well depths, well widths and barrier widths, and may provide us with some useful information for the design and improvement of microelectronic MQW devices, such as LEDs, solar cells, laser diodes and so on.

\section{REFERENCES}

[1] Ü. Özgür, H. Y. Liu, X. Li, X. F. Ni and H. Morkoç, Proceedings of the IEEE, Vol. 98, 2010, pp. 1180-1196.

[2] J. R. Xu, M. F. Schubert, A. N. Noemaun, D. Zhu, J. K. Kim, E. F. Schubert, M. H. Kim, H. J. Chung, S. Yoon, C. Sone and Y. Park, Applied Physics Letters, Vol. 94, 2009, Article ID: 011113. doi:10.1063/1.3058687
[3] M. H. Kim, M. F. Schubert, Q. Dai, J. K. Kim, E. F. Schubert, J. Piprek and Y. Park, Applied Physics Letters, Vol. 91, 2007, Article ID: 183507. doi: $10.1063 / 1.2800290$

[4] H. Morkoç, "Handbook of Nitride Semiconductors and Devices," Vol. 3, John Wiley \& Sons Inc., New York, 2008.

[5] M. F. Schubert, et al., Applied Physics Letters, Vol. 91, 2007, Article ID: 231114. doi:10.1063/1.2822442

[6] M. F. Schubert, J. Xu, J. K. Kim, E. F. Schubert, M. H. Kim, S. Yoon, S. M. Lee, C. Sone, T. Sakong and Y. Park, Applied Physics Letters, Vol. 93, 2008, Article ID: 041102. doi:10.1063/1.2963029

[7] M. R. Krames, O. B. Shchekin, R. Mueller-Mach, G. O. Mueller, L. Zhou, G. Harbers and M. G. Craford, Journal of Display Technology, Vol. 3, 2007, pp. 160-175. doi:10.1109/JDT.2007.895339

[8] X. Ni, Q. Fan, R. Shimad, Ü. Özgür and H. Morkoç, Applied Physics Letters, Vol. 93, 2008, Article ID: 171113. doi:10.1063/1.3012388

[9] J. Xie, X. Ni, Q. Fan, R. Shimada, Ü. Özgür and H. Morkoç, Applied Physics Letters, Vol. 93, 2008, Article ID: 121107. doi:10.1063/1.2988324

[10] S.-H. Han, D.-Y. Lee, H.-W. Shim, G.-C. Kim, Y. S. Kim, S.-T. Kim, S.-J. Lee, C.-Y. Cho and S.-J. Park, Journal of Physics D: Applied Physics, Vol. 43, 2010, Article ID: 354004. doi:10.1088/0022-3727/43/35/354004

[11] S. Nakamura, M. Senoh, S.-I. Nagahama, N. Iwasa, T. Yamada, T. Matsushita, H. Kiyoku and Y. Sugimoto, Japanese Journal of Applied Physics, Vol. 35, 1996, pp. L74-L76. doi:10.1143/JJAP.35.L74

[12] R. Dahal, B. Pantha, J. Li, J. Y. Lin and H. X. Jiang, Applied Physics Letters, Vol. 94, 2009, Article ID: 063505. doi:10.1063/1.3081123

[13] K. W. J. Barnham, I. Ballard, J. P. Connolly, N. J. EkinsDaukes, B. G. Kluftinger, J. Nelson and C. Rohr, Physica E, Vol. 14, 2002, pp. 27-36. doi:10.1016/S1386-9477(02)00356-9

[14] P.-F. Yuh and K. L. Wang, Physical Review B, Vol. 38, 1988, pp. 13307-13315.

[15] S. Mishra and S. Satpathy, Physical Review B, Vol. 68, 2003, Article ID: 045121.

[16] M. Barbier, P. Vasilopoulos and F. M. Peeters, Physical Review B, Vol. 82, 2010, Article ID: 235408. doi:10.1103/PhysRevB.82.235408

[17] C. Kittles, "Introduction to Solid State Physics," 8th Edition, John Wiley \& Sons, Inc., New York, 2004.

[18] R. Dahal, J. Li, K. Aryal, J. Y. Lin and H. X. Jiang, Applied Physics Letters, Vol. 97, 2010, Article ID: 073115. doi:10.1063/1.3481424

[19] Y.-K. Kuo, J.-Y. Chang, M.-C. Tsai and S.-H. Yen, Applied Physics Letters, Vol. 95, 2009, Article ID: 011116. doi:10.1063/1.3176406 